\title{
Low magnetic field anomaly of the Hall effect in disordered 2D systems: Interplay between weak localization and electron-electron interaction
}

\author{
G. M. Minkov, ${ }^{1,2}$ A. V. Germanenko, ${ }^{2}$ O. E. Rut, ${ }^{2}$ A. A. Sherstobitov, ${ }^{1,2}$ and B. N. Zvonkov ${ }^{3}$ \\ ${ }^{1}$ Institute of Metal Physics RAS, 620219 Ekaterinburg, Russia \\ ${ }^{2}$ Ural State University, 620083 Ekaterinburg, Russia \\ ${ }^{3}$ Physical-Technical Research Institute, University of Nizhni Novgorod, 603600 Nizhni Novgorod, Russia
}

(Dated: June 4, 2022)

\begin{abstract}
The nonlinear behavior of the Hall resistivity at low magnetic fields in single quantum well $\mathrm{GaAs} / \mathrm{In}_{x} \mathrm{Ga}_{1-x} \mathrm{As} / \mathrm{GaAs}$ heterostructures with degenerated electron gas is studied. It has been found that this anomaly is accompanied by the weaker temperature dependence of the conductivity as compared with that predicted by the first-order theory of the quantum corrections to the conductivity. We show that both effects in strongly disordered systems stem from the second order quantum correction caused by the effect of weak localization on the interaction correction and vice versa. This correction contributes mainly to the diagonal component of the conductivity tensor, it depends on the magnetic field like the weak localization correction and on the temperature like the interaction contribution.
\end{abstract}

\section{INTRODUCTION}

The quantum corrections to the conductivity, namely the interference or weak localization (WL) correction and correction due to electron-electron $(e-e)$ interaction, wholly determine the temperature and magnetic field dependences of the conductivity $(\sigma)$ at $T \ll E_{F}, \tau^{-1}$, where $E_{F}$ and $\tau$ are the Fermi energy and the transport relaxation time, respectively (hereafter we set $k_{B}=\hbar=1$ for brevity) $\frac{1}{\underline{*}}$ The modern theory being elaborated since $1980^{2} \underline{-6}$ allows ones to describe most of experimental results obtained on the well controlled semiconductor twodimensional systems quantitatively. However, one peculiarity, namely the low magnetic field dependence of the Hall coefficient $\left(R_{H}\right)$, referred as beak in what follows, remains a puzzle. The magnetic field scale of the beak is close to the transport magnetic field $B_{t r}=\hbar / 2 e l^{2}$, where $l$ is the mean free path, i.e., close to the field, in which the main part of the interference correction is suppressed. As a rule, the Hall coefficient increases in absolute value with the growing magnetic field, and the magnitude of the beak is close to that of the negative magnetoresistivity caused by suppression of the weak localization: $\left|\delta R_{H} / R_{H}\right| \sim\left|\delta \sigma^{W L}\right| / \sigma$. The existence of low field anomaly in $R_{H}$ was pointed out in the pioneering papers on the quantum corrections $\underline{7-9}^{\underline{\underline{9}}}$ In the later papers the anomaly of $R_{H}$ behavior is not mentioned, although the beak is observed practically in all the $2 \mathrm{D}$ structures $\underline{\underline{10}-12}$

Theories of the weak localization and interaction correction do not predict any low magnetic field dependence of the Hall coefficient. The WL theory asserts that the quantum interference renormalizes the transport relaxation time and, consequently, does not lead to correction in the Hall coefficient. The $e$-e interaction within the diffusion regime, $T \tau \ll 1$, contributes to the longitudinal conductivity $\sigma_{x x}$ only and this correction does not depend on the magnetic field while the Zeeman splitting is less than the temperature, $|g| \mu_{B} B<T$. So, this cor- rection leads to the temperature dependence of the Hall coefficient, in the magnetic field $R_{H}$ remains constant $\underline{13}$ Thus, the origin of the beak in the $B$ dependence of the Hall coefficient remains enigmatic.

We have analyzed numerous experimental data regarding the low field anomaly of the Hall coefficient for more than thirty $\mathrm{GaAs} / \mathrm{In}_{x} \mathrm{Ga}_{1-x} \mathrm{As} / \mathrm{GaAs}$ and $\mathrm{Al}_{x} \mathrm{Ga}_{1-x} \mathrm{As} / \mathrm{GaAs} / \mathrm{Al}_{x} \mathrm{Ga}_{1-x}$ As structures both with the electron and hole $2 \mathrm{D}$ gas with the carrier density from $1 \times 10^{11} \mathrm{~cm}^{-2}$ to $2 \times 10^{12} \mathrm{~cm}^{-2}$ and the mobility from $1 \times 10^{2} \mathrm{~cm}^{2} / \mathrm{Vs}$ to $2 \times 10^{4} \mathrm{~cm}^{2} / \mathrm{Vs}$. We have not found any correlation between the beak magnitude and such the structure parameters as the transport and quantum relaxation time, carries density, spin-orbit interaction strength and so on. It indicates in our opinion that there is no universal reason for such a behavior of the Hall coefficient. However, we believe that in strongly disordered structures in deep diffusion regime the origin of the beak in the $R_{H}$ vs $B$ dependence is clear. In this paper we show that it comes from the interplay between the weak localization and interaction effects. This interplay term contributes to $\sigma_{x x}$ only like the interaction correction and depends on the magnetic field like the WL correction.

\section{EXPERIMENTAL DETAILS}

The structures investigated were grown by metalorganic vapor-phase epitaxy on a semiinsulating GaAs substrate and consist of $0.5-\mu \mathrm{m}$-thick undoped GaAs epilayer, a $\operatorname{In}_{x} \mathrm{Ga}_{1-x}$ As quantum well with $\mathrm{Sn}$ or $\mathrm{Si} \delta$ layer situated in the well center and a $200 \mathrm{~nm}$ cap layer of undoped GaAs. The samples were mesa etched into standard Hall bars and then an $\mathrm{Al}$ gate electrode was deposited by thermal evaporation onto the cap layer through a mask. Varying the gate voltage $\left(V_{g}\right)$ we were able to change the electron density $(n)$ and the conductivity of $2 \mathrm{D}$ electron gas in the quantum well. We studied samples prepared from four wafers with different well 


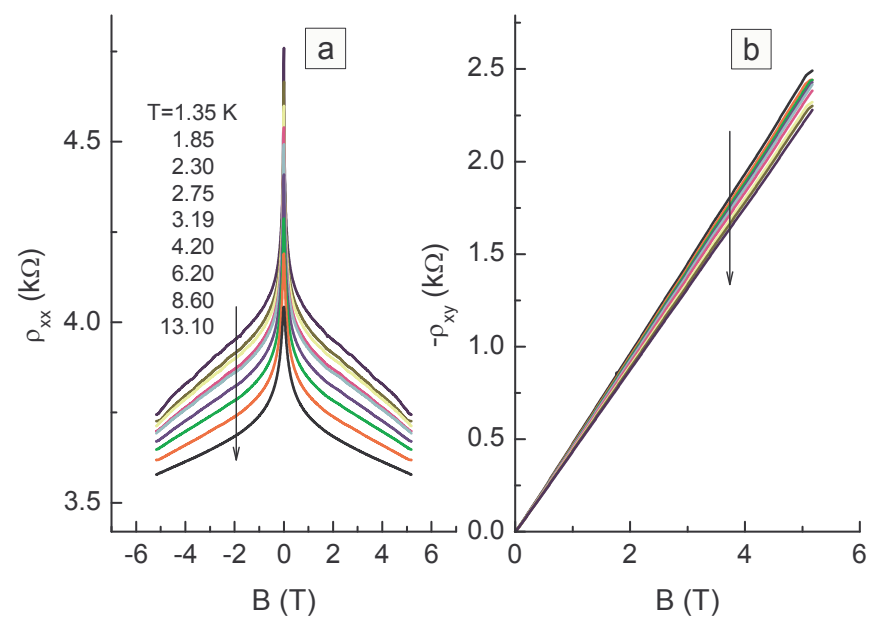

FIG. 1. (Color online) The magnetic field dependences of $\rho_{x x}$ (a) and $\rho_{x y}$ (b) for $V_{g}=-1 \mathrm{~V}$ taken at different temperatures. The arrows indicate the temperature growth.

width, doping level and well composition. All the measurements were carried out in the Ohmic regime using DC technique. The results obtained were mostly analogous, therefore we will discuss the results for the structure 4261 studied more thoroughly. The quantum well width in this structure is $8 \mathrm{~nm}$, indium content in the quantum well is 0.2 and tin density in $\delta$ layer is about $2 \times 10^{12} \mathrm{~cm}^{-2}$.

\section{RESULTS AND DISCUSSION}

Let us consider the magnetic field dependences of $\rho_{x x}$ and $\rho_{x y}$ for $V_{g}=-1 \mathrm{~V}$ (Fig. (1) taken at different temperatures. These dependences are typical for such a type of systems. The sharp negative magnetoresistance at low magnetic field [Fig. 1(a)] results from suppression of the WL contribution. A crossover to the parabolic-like behavior of $\rho_{x x}$ at $B \gtrsim 2 \mathrm{~T}$ and the decrease of $\rho_{x y}$ with the temperature increase come from the $e$-e interaction correction. For the first sight, $\rho_{x y}$ linearly depends on the magnetic field [Fig. 1(b)] as predicted theoretically. Let us, however, inspect the Hall coefficient, $R_{H}=\rho_{x y} / B$, which magnetic field dependences taken for different gate voltages at $T=1.4 \mathrm{~K}$ are plotted in Fig. 2(a). It is evident that $R_{H}$ decreases in magnitude when $B$ goes to zero for all the gate voltages. Comparing these dependences with that for magnetoresistance [presented in Fig. 2(b)], one can see that the characteristic scales in $B$ domain for the $R_{H}$ beak and for the interference induced negative magnetoresistance are close; the main changes happen at $B \lesssim B_{t r}$ in both cases. Therefore, before to discuss the low field peculiarity of the Hall coefficient let us analyze the contributions of the interference and interaction.

First we remind the reader of the basic results of the quantum correction theory that will be used for analysis. The expression for the conductivity tensor components

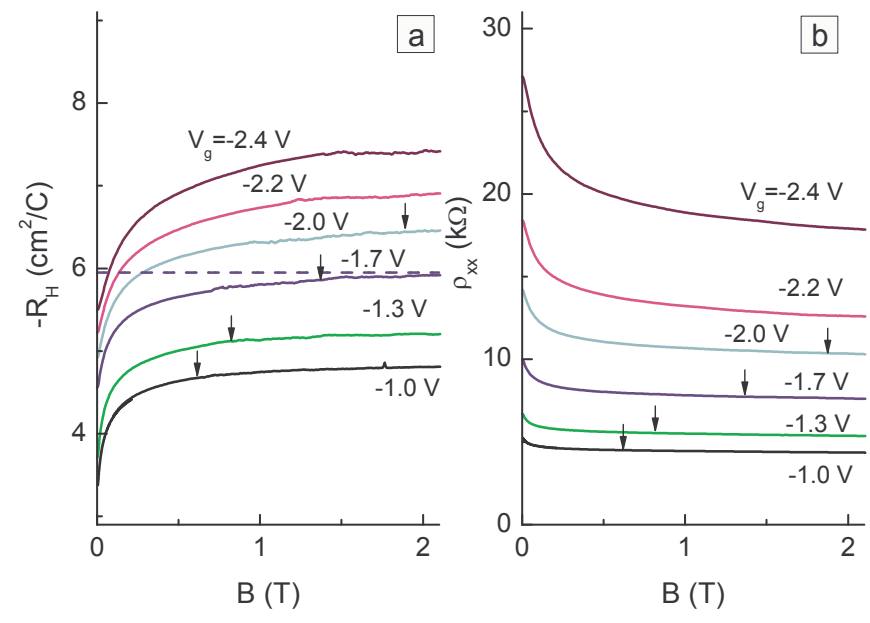

FIG. 2. (Color online) The magnetic field dependences of $R_{H}$ (a) and $\rho_{x x}$ (b) taken for different gate voltages at $T=1.4 \mathrm{~K}$. The dashed line is the Hall constant for $V_{g}=-1.7 \mathrm{~V}$ obtained from the linear interpolation of $\rho_{x y}$ within the low magnetic field domain, $|B| \lesssim 5 B_{t r}$. The arrows indicates the $B_{t r}$ values.

taking into account the first order in $\delta \sigma / \sigma$ corrections are the following: 14

$$
\begin{aligned}
\sigma_{x x}(B, T) & =\frac{\operatorname{en} \mu(B, T)}{1+[\mu(B, T) B]^{2}}+\delta \sigma_{x x}^{e e}(T), \\
\sigma_{x y}(B, T) & =\frac{\operatorname{en} \mu(B, T)^{2} B}{1+[\mu(B, T) B]^{2}} .
\end{aligned}
$$

In the actual case of $T \tau \ll 1$, the correction $\delta \sigma_{x x}^{e e}$ is just the Altshuler-Aronov (AA) correction given by $2,15-19$

$$
\delta \sigma^{\mathrm{AA}}(T)=K_{e e}^{\mathrm{AA}} G_{0} \ln (T \tau),
$$

where

$$
K_{e e}^{\mathrm{AA}}=1+3\left[1-\frac{1+\gamma_{2}}{\gamma_{2}} \ln \left(1+\gamma_{2}\right)\right]
$$

with $G_{0}=e^{2} / \pi h$ and $\gamma_{2}$ standing for the Landau's Fermi liquid amplitude. Because the WL correction is reduced to the renormalization of the transport relaxation time, $\stackrel{20}{=}$ it is incorporated in Eqs. (1) and (2) into the mobility $\mu$ in such a way that

$$
\delta \sigma^{W L}(B, T)=e n \delta \mu(B, T),
$$

where

$$
\delta \sigma^{W L}(0, T)=G_{0} \ln \left[\frac{\tau}{\tau_{\phi}(T)}\right],
$$

and $\Delta \sigma^{W L}(B, T)=\delta \sigma^{W L}(B, T)-\delta \sigma^{W L}(0, T)$ is described by the expression ${ }^{21,22}$

$$
\begin{aligned}
\Delta \sigma^{W L}(B, T) & =\alpha G_{0} \mathcal{H}\left(\frac{\tau}{\tau_{\phi}(T)}, \frac{B}{B_{t r}}\right) \\
\mathcal{H}(x, y) & =\psi\left(\frac{1}{2}+\frac{x}{y}\right)-\psi\left(\frac{1}{2}+\frac{1}{y}\right)-\ln x
\end{aligned}
$$



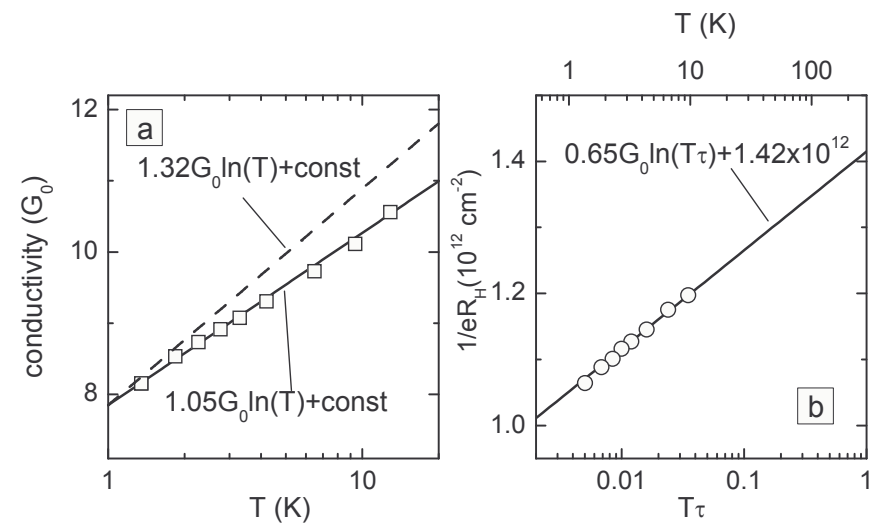

FIG. 3. (Color online) The temperature dependence of $\sigma$ at $B=0$ (a) and $\left[e R_{H}(B=4 \mathrm{~T})\right]^{-1}$ (b) for $V_{g}=-1.7 \mathrm{~V}$ $\left(\tau=2.7 \times 10^{-14} \mathrm{~s}\right)$.

Here, $\tau_{\phi}$ is the phase relaxation time, $\psi(x)$ is a digamma function, and $\alpha$ is the prefactor, whose value depends on the conductivity if one takes into account two-loop localization correction and the interplay of the weak localization and interaction,,$\frac{23}{,}$

$$
\alpha \simeq 1-\frac{2 G_{0}}{\sigma}, \quad \sigma<2 G_{0}
$$

We turn now to the analysis of the data. By way of example we consider the case of $V_{g}=-1.7 \mathrm{~V}$. As seen from Fig. 3(a) the temperature dependence of the conductivity without magnetic field is close to the logarithmic one, $\sigma(T)=\beta \ln \left(T / T_{0}\right)$, with the slope $\beta$ equal to $1.05 \pm 0.05$. To find what portion of the slope comes from WL let us inspect the low field magnetoconductivity [Fig. 4(a)]. The electron density $n=(1.42 \pm 0.03) \times 10^{12} \mathrm{~cm}^{-2}$ needed for the analysis we obtain from the extrapolation of the temperature dependence of the Hall density $n=1 / e R_{H}$ taken at high magnetic field, $B=4 \mathrm{~T}$, to $T \tau=1$ [Fig. [3(b)]. Such a dependence of $R_{H}$ comes from the diffusion contribution of the interaction, which vanishes at $T \tau=1$. So, the value of $1 / e R_{H}$ at $T \tau=1$ actually gives the electron density. An analysis shows that Eq. (7) the experimental dependences $\Delta \sigma(B)=1 / \rho_{x x}(B)-1 / \rho_{x x}(0)$ [see Fig. [(a)]. The values of $\tau_{\phi}$ and $\alpha$ found from the fit within magnetic field range $|B|<0.2 B_{t r}$ for different temperatures are plotted in Figs. 4 (b) and 4 (c), respectively. One can see that $\tau_{\phi}(T)$ is very close to $1 / T$. The prefactor values being noticeably less than unity, $\alpha=0.6 \ldots 0.7$, decreases slightly with the decreasing conductivity. As Fig. 4(c) shows, such a behavior agrees well with the theoretical result, Eq. (8). So, the value of $\tau_{\phi}$ found from the fit of Eq. (77) to the data is the value of the phase breaking time. As mentioned above it is inversely proportional to the temperature in whole agreement with theoretical prediction. ${ }^{2}$ Thus, taking into account Eq. (6) we conclude that the weak localization gives the unit in the slope of the $\sigma$ vs $\ln T$ dependence at $B=0$.

Let us determine now the interaction contribution to the conductivity. One can find it from the temperature
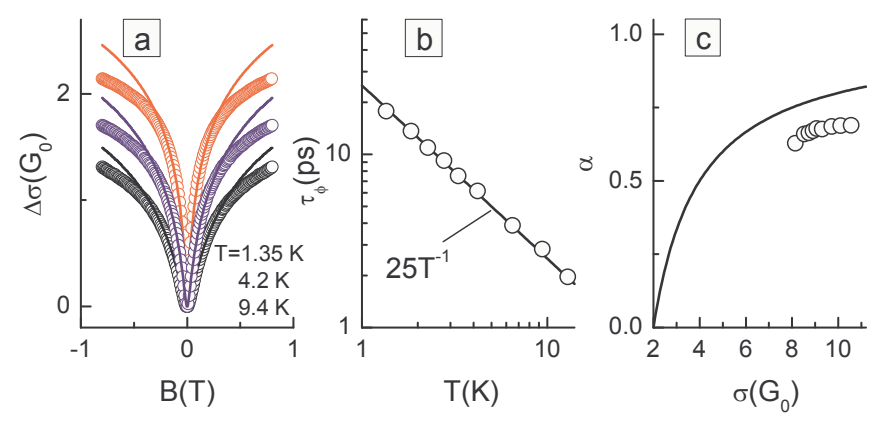

FIG. 4. (Color online) (a) The magnetic field dependence of $\Delta \sigma$ for different temperatures. Symbols are the experimental data, lines are the results of the best fit by Eq. (7) within the range of $B$ from $-0.2 B_{t r}$ to $0.2 B_{t r}$, where $B_{t r}=1.26 \mathrm{~T}$. (b) The temperature dependences of $\tau_{\phi}$ found from the fit. (c) The prefactor $\alpha$ plotted as a function of the conductivity at $B=0$ driven by the temperature. The solid line is Eq. (8).

dependence of the Hall coefficient at high magnetic field [see Fig. [3(b)] because $\delta R_{H} / R_{H} \simeq-2 \delta \sigma_{x x}^{e e} / \sigma_{0}$ under the condition $\left|\delta \sigma_{x x}^{e e}\right| \ll \sigma_{0}$. This gives $\delta \sigma_{x x}^{e e} \simeq 0.32 \ln T \tau$. However, the more straightforward way (which does not require the fulfilment of this condition) is the following 24 Since the interaction in the diffusion regime contributes to $\sigma_{x x}$ only, one should find such the contribution to the conductivity which exists in $\sigma_{x x}$ but is absent in $\sigma_{x y}$. It can be done by expressing $\mu(B, T)$ from Eq. (2) and substituting it in Eq.(10). Doing so we obtain the expression

$$
\delta \sigma_{x x}^{e e}=\frac{1}{\rho_{x x}^{2}+\rho_{x y}^{2}}\left[\rho_{x x}-\rho_{x y} \sqrt{\frac{e n\left(\rho_{x x}^{2}+\rho_{x y}^{2}\right)}{\rho_{x y} B}-1}\right]
$$

that allows us to find $\delta \sigma_{x x}^{e e}$ using the experimental quantities $\rho_{x x}$ and $\rho_{x y}$. The magnetic field dependences of $\delta \sigma_{x x}^{e e}$ found by this way at different temperatures are presented in Fig. [5(a). One can see that $\delta \sigma_{x x}^{e e}$ is practically independent of the magnetic field while $B \gtrsim(1.5-2) \mathrm{T}$. The temperature dependence of $\delta \sigma_{x x}^{e e}$ is logarithmic, and the slope $K_{e e}^{\exp }$ being equal to $0.32 \pm 0.05$ remains independent of the magnetic field at $B \gtrsim 2 \mathrm{~T}$ [see Figs. 5 (b) and 5(c)]. Such the behavior agrees well with Eq. (3) and, thus, $K_{e e}^{\exp }=0.32 \pm 0.05$ is just the value of $K_{e e}^{\mathrm{AA}}$. Using Eq. (4) one obtains $\gamma_{2} \simeq 0.53$ that is in a good agreement with the results of Ref. 4 if one takes into account the renormalization effect. 25,26 Thus, the $T$ dependence of $\delta \sigma_{x x}^{e e}$ in high magnetic field, $B \gtrsim(1.5-2) \mathrm{T}$, is determined by the AA quantum correction.

As we have obtained the value of $K_{e e}^{\mathrm{AA}}$ responsible for the AA contribution, we can compare the value of $\beta$ describing the experimental $T$ dependence of $\sigma$ at $B=0$ with the value of $1+K_{e e}^{\mathrm{AA}}$ (recall that 1 comes from the WL effect) found from the analysis of the data in the magnetic field. If the model used and, consequently, Eqs. (11)-(8), are correct, the values of $1+K_{e e}^{\mathrm{AA}}$ and $\beta$ should be equal to each other. We have that $\beta=1.05 \pm 0.05$ [see Fig. 3(a)] is visibly less than 


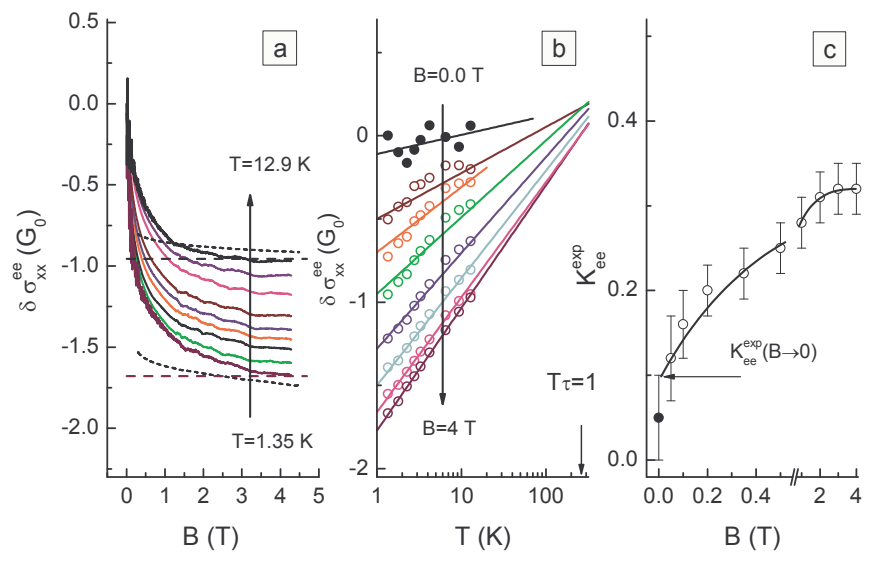

FIG. 5. (Color online) (a) The magnetic field dependences of the $\delta \sigma_{x x}^{e e}$ for different temperatures: $T=1.35,1.8,2.27,2.77$, $3.3,4.2,6.5,9.4$, and $12.9 \mathrm{~K}$. The dashed lines are $K_{e e}^{\mathrm{AA}} \ln T \tau$ for $T=1.35 \mathrm{~K}$ and $12.9 \mathrm{~K}$ with $\tau=2.7 \times 10^{-14} \mathrm{~s}$ and $K_{e e}^{\mathrm{AA}}=0.32$ found from the $T$ dependence of $R_{H}$ in high magnetic field [see Fig. 3(b)]. The dotted lines are $\delta \sigma_{x x}^{e e}$ obtained for $T=1.35 \mathrm{~K}$ and $12.9 \mathrm{~K}$ in the assumption that $R_{H}$ is independent of $B$. (b) The temperature dependence of $\delta \sigma_{x x}^{e e}$ for different magnetic field: $B=0,0.05,0.1,0.2,0.5$, $1.0,2.0$, and $4.0 \mathrm{~T}$. Data at $B=0$ are obtained by extrapolation of $\delta \sigma_{x x}^{e e}$ vs $B$ curves shown in the panel (a) to $B=0$ as described on page 5. (c) The $B$-dependence of the slope $K_{e e}^{\exp }$ of the $\delta \sigma_{x x}^{e e}$ vs $\ln T$ dependences shown in the panel (b).

$1+K_{e e}^{\mathrm{AA}}=1.32 \pm 0.05$. The reason for this discrepancy is transparent. The $K_{e e}^{\mathrm{AA}}$ value has been above obtained at relatively strong magnetic field, in which the interaction contribution does not depend on the magnetic field as the theory predicts. However, inspection of Fig. 5 shows that not only $\delta \sigma_{x x}^{e e}$ diminishes in absolute value at $B \rightarrow 0$ but the slope $K_{e e}^{\exp }$ decreases as well. Of course, the accuracy of $K_{e e}^{\exp }$ determination is not very high in low magnetic field. As seen from Fig. 5 (a) the experimental $\delta \sigma_{x x}^{e e}$ vs $B$ plots are very noisy near $B=0$. The reason is very clear. The expression, Eq. (9), used for the data treatment contains $B$ in the denominator. Nevertheless, extrapolating the $K_{e e}^{\exp }$ vs $B$ data to $B=0$ one obtains $K_{e e}^{\exp }(B \rightarrow 0)=0.1 \pm 0.05$. Together with 1 coming from the WL effect we have the value, which practically coincides with $\beta=1.05 \pm 0.05$.

In principle, the $K_{e e}^{\exp }$ change could be induced by the $K_{e e}^{\mathrm{AA}}$ decrease due to suppression of two of three triplet channels in Eq. (3) due to the Zeeman effect, 17,19,27-29 However, this effect is negligible in our case due to the low value of the effective $g$-factor, $g \sim 0.5$. Moreover, if the Zeeman splitting would be important, the $T$ dependence of $\delta \sigma_{x x}^{e e}$ in high magnetic field should be strongly nonlogarithmic as it takes place in 2D hole gas (see Fig. 2 in Ref. 29).

It is essential to note that the strong decrease of $\delta \sigma_{x x}^{e e}$ in absolute value with lowering magnetic filed results from the beak in $R_{H}$ vs $B$ dependence. Really, if one uses the linear interpolation of $\rho_{x y}$ within the range $\pm(4-5) B_{t r}$ in the above procedure, i.e., one supposes that $R_{H}$ is con-
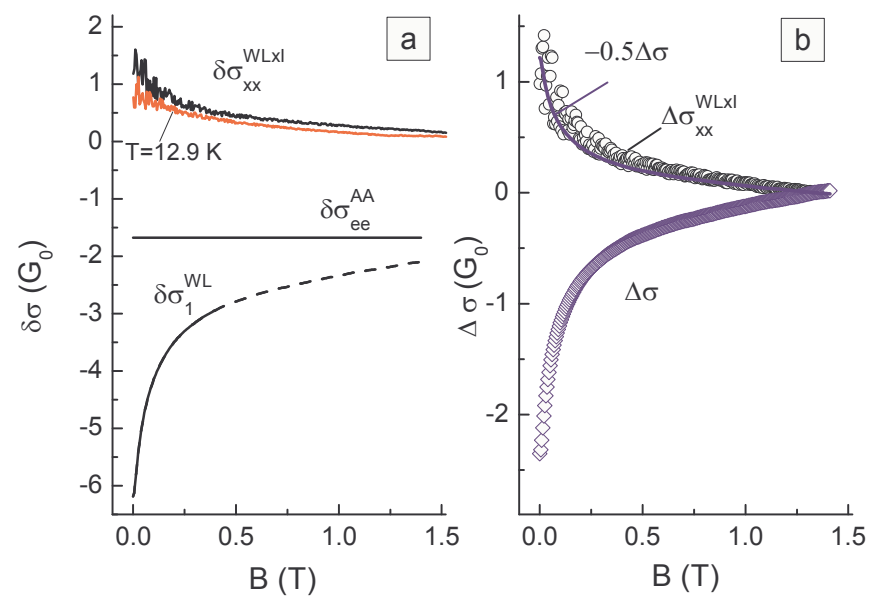

FIG. 6. (Color online) (a) The magnetic field dependences of $\delta \sigma_{1}^{\mathrm{WL}}, \delta \sigma_{e e}^{\mathrm{AA}}$ and $\delta \sigma_{x x}^{\mathrm{WL} \times \mathrm{I}}$ for $T=1.35 \mathrm{~K}$. The dashed line indicates that the estimate for $\delta \sigma_{1}^{\mathrm{WL}}$ is rough in this range, because Eq. (10) is valid when $B \ll B_{t r}$ (b) The experimental dependences $\Delta \sigma=\rho_{x x}^{-1}(B)-\rho_{x x}^{-1}\left(B_{t r}\right)$ and $\Delta \sigma_{x x}^{\mathrm{WL} \times \mathrm{I}}=\delta \sigma_{x x}^{\mathrm{WL} \times \mathrm{I}}(B)-\delta \sigma_{x x}^{\mathrm{WL} \times \mathrm{I}}\left(B_{t r}\right)$.

stant as shown in Fig. 2(b) for $V_{g}=-1.7 \mathrm{~V}$ by the dashed line, we obtain $\delta \sigma_{x x}^{e e}$, which is practically independent of the magnetic field [dotted lines in Fig. 5(a)].

Thus, there is common reason behind the beak in the $R_{H}$ vs $B$ dependence and the existence of magnetic field dependence of $\delta \sigma_{x x}^{e e}$. Because the $T$ and $B$ changes of the conductivity are rather large $(\delta \sigma$ is about $(20-30) \%$ of $\sigma)$, it is natural to assume that the second order corrections play an important role under our conditions.

The role of the second order effects is studied in the number of paper $\stackrel{3,23,30}{-35}$ The second-order interaction correction (not involving Cooperons), $\delta \sigma_{2}^{\mathrm{I}}$, logarithmically depends on the temperature, but does not depend on the magnetic field analogously to the AA correction 34.35 That is why it barely gives the correction to $K_{e e}^{\mathrm{AA}}$, Eq. (41), and does not affect the low magnetic field magnetoresistance $\Delta \sigma(B)$. The other two second order terms have an impact on $\Delta \sigma(B)$. They are $\delta \sigma^{\mathrm{WL} \times \mathrm{I}}$ coming from the interplay between the weak localization and the interaction effects,$\frac{3}{,}$ and $\delta \sigma_{2}^{\mathrm{WL}}$, which is the second order interference correction. ${ }^{23}$ Except for opposite sign the magnetic field dependences of both terms are close to that for the first order interference correction. Namely this fact results in the appearance of $\alpha<1$ in Eq. (77). Science the interference correction stems from the ( $B$-dependent) correction to the impurity scattering cross section and hence renormalizes the value of the elastic transport scattering rate $1 / \tau$, the higher order interference corrections do not contribute to the Hall effect $\frac{36,37}{3}$ analogously to the first order one. $\stackrel{2}{=}$ Moreover, $\delta \sigma_{2}^{\mathrm{WL}}$ does not contribute to the $T$ dependence of $\sigma$ at zero magnetic field, since the terms of the second and third orders cancel out in the interference correction $\underline{30,31}$ Thus, it is reasonable to assume that the main effect comes from the interplay term $\delta \sigma^{\mathrm{WL} \times \mathrm{I}}$. 
Generally, the interplay effect may give corrections to both components of the conductivity tensor. We designate them as $\delta \sigma_{x x}^{\mathrm{WL} \times \mathrm{I}}$ and $\delta \sigma_{x y}^{\mathrm{WL} \times \mathrm{I}}$. Because $\sigma_{x y}^{\mathrm{WL} \times \mathrm{I}}=0$ at $B=0$, the difference between $\beta$ and $1+K_{e e}^{\mathrm{AA}}$ results from $\delta \sigma_{x x}^{\mathrm{WL} \times \mathrm{I}}$. Suppose that $\delta \sigma_{x y}^{\mathrm{WL} \times \mathrm{I}}$ is small in the presence of magnetic field as well: $\delta \sigma_{x y}^{\mathrm{WL} \times \mathrm{I}} \ll \mu B \delta \sigma_{x x}^{\mathrm{WL} \times \mathrm{I}}$. In this case the quantity $\delta \sigma_{x x}^{e e}$ found above is just the sum of the AA correction, which is independent of the magnetic field and logarithmically dependent on the temperature, and the second order correction $\delta \sigma_{x x}^{\mathrm{WL} \times \mathrm{I}}$, which depends both on $T$ and $B: \delta \sigma_{x x}^{e e}=\delta \sigma_{e e}^{\mathrm{AA}}(T)+\delta \sigma_{x x}^{\mathrm{WL} \times \mathrm{I}}(B, T)$. In Fig. 6(a), the value of $\delta \sigma_{x x}^{\mathrm{WL} \times \mathrm{I}}$ found as $\delta \sigma_{x x}^{e e}-K_{e e}^{\mathrm{AA}} \ln T \tau$ with $\tau=2.7 \times 10^{-14} \mathrm{~s}$ and $K_{e e}^{\mathrm{AA}}=0.32$ are plotted against the magnetic field. For comparison, the one-loop WL corrections $\delta \sigma_{e e}^{\mathrm{AA}}(T)$ and $\delta \sigma_{1}^{\mathrm{WL}}$ are depicted in the same figure. The $\delta \sigma_{1}^{\mathrm{WL}}$ correction is obtained from the experimental data in accordance with Eqs. (6) and (7) as follows

$$
\delta \sigma_{1}^{\mathrm{WL}} \simeq \frac{\Delta \sigma(B)}{\alpha}-\ln \left(\frac{\tau_{\phi}}{\tau}\right) .
$$

Two important properties of $\delta \sigma_{x x}^{\mathrm{WL} \times \mathrm{I}}$ are evident. First, the interplay correction is metallic-like in contrast to the WL and AA corrections, i.e., it increases with temperature decrease [see Fig. 6(a)]. Qualitatively, this explains the difference between $1+K_{e e}^{\mathrm{AA}}$ and $\beta$. Second, the $B$ range, where the main changes in $\delta \sigma_{x x}^{\mathrm{WL} \times \mathrm{I}}$ occur, is the same as for $\delta \sigma_{1}^{\mathrm{WL}}: B<B_{t r} \simeq 1.36 \mathrm{~T}$. The fact that $\delta \sigma_{x x}^{\mathrm{WL} \times \mathrm{I}}$ vs $B$ curve is close in the shape to the low magnetic field magnetoconductance is illustrated in Fig. 6(b), where the dependences $\Delta \sigma(B)=\rho_{x x}^{-1}(B)-\rho_{x x}^{-1}\left(B_{t r}\right)$ and $\Delta \sigma_{x x}^{\mathrm{WL} \times \mathrm{I}}(B)=\delta \sigma_{x x}^{\mathrm{WL} \times \mathrm{I}}(B)-\delta \sigma_{x x}^{\mathrm{WL} \times \mathrm{I}}\left(B_{t r}\right)$ are shown. As seen $\Delta \sigma(B)$ multiplied by the factor $\gamma$ of -0.5 fits the $\Delta \sigma_{x x}^{\mathrm{WL} \times \mathrm{I}}$ dots rather well.

The second property has been used to obtain the value of $\delta \sigma_{x x}^{\mathrm{WL} \times \mathrm{I}}(B=0)$ [and $\delta \sigma_{x x}^{e e}(B=0)$ shown in Fig. 5 (b) by solid circles]. We have interpolated the experimental $B$ dependences of $\delta \sigma_{x x}^{\mathrm{WL} \times \mathrm{I}}$ (and $\delta \sigma_{x x}^{e e}$ ) at $B<B_{t r}$ by the experimental curve $\Delta \sigma(B)$ excluding the noisy vicinity of $B=0$ and interpolated it to $B=0$. The $T$ dependence of $\delta \sigma_{x x}^{\mathrm{WL} \times \mathrm{I}}(0)$ found in such a way is close to the logarithmic one with the slope $K^{\mathrm{WL} \times \mathrm{I}}=-0.3 \pm 0.1$.

Thus, the temperature dependence of the conductivity at $B=0$ caused by all three contributions $\delta \sigma_{1}^{\mathrm{WL}}, \delta \sigma_{e e}^{\mathrm{AA}}$, and $\delta \sigma_{x x}^{\mathrm{WL} \times \mathrm{I}}$ is very close to that observed experimentally. The total slope equal to $1+K_{e e}^{\mathrm{AA}}+K^{\mathrm{WL} \times \mathrm{I}}=1.02 \pm 0.1$ is in a good agreement with the experimental value $\beta=$ $1.05 \pm 0.05$ [see Fig. 3(a)].

Analysis described has been performed within wide range of the conductivity driven by the gate voltage. The values of $K^{\mathrm{WL} \times \mathrm{I}}$ plotted against $\sigma$ at $T=1.35 \mathrm{~K}$ are shown in Fig. 7(a). In the same figure the difference between $1+K_{e e}^{\mathrm{AA}}$ and $\beta$ is depicted. It is seen that both data are close to each other at low conductivity, $\sigma<20 G_{0}$. At higher conductivity, they diverge drastically.

The contribution of $\delta \sigma_{x x}^{\mathrm{WL} \times \mathrm{I}}$ to the magnetoconductivity is illustrated by Fig. [7(b). We characterize it by the product $\gamma \alpha$ which is $\Delta \sigma_{x x}^{\mathrm{WL} \times \mathrm{I}}(B)$ to $\Delta \sigma_{1}^{\mathrm{WL}}(B)$ ra-
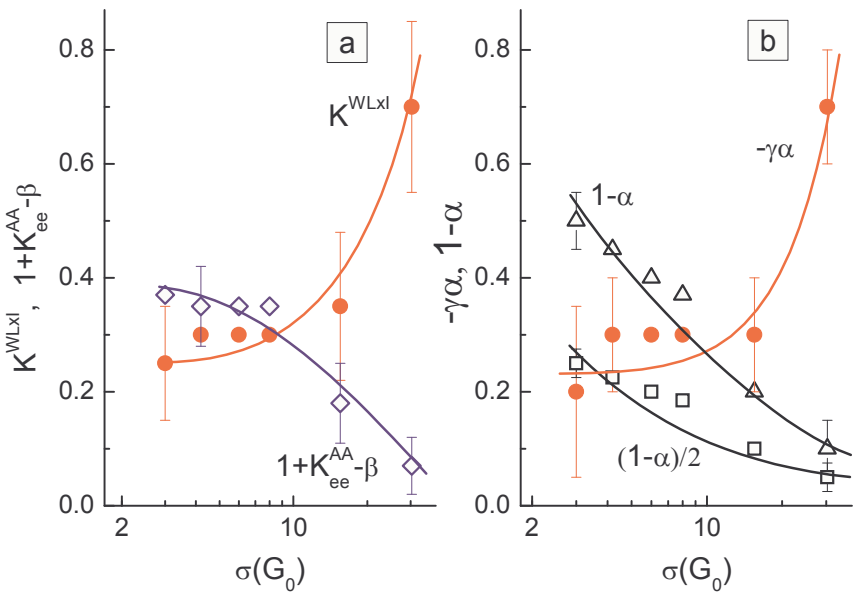

FIG. 7. (Color online) (a) The values of $K^{\mathrm{WL} \times \mathrm{I}}$ (circles) and the difference between $1+K_{e e}^{\mathrm{AA}}$ and $\beta$ (diamonds) as a function of the conductivity at $T=1.35 \mathrm{~K}$. (b) The conductivity dependence of $-\gamma \alpha$ (circles), $1-\alpha$ (triangles), and $(1-\alpha) / 2$ (squares). Lines are provided as a guide to the eye. The conductivity in both panels is driven by the gate voltage.

tio. If one supposes that $\delta \sigma_{x x}^{\mathrm{WL} \times \mathrm{I}}$ is alone and there is no $\Delta \sigma_{2}^{\mathrm{WL}}$ contribution to the magnetoconductivity, this value should be equal to $1-\alpha$. If these correction are the same in magnitude (as it turns out theoretically for the short-range interaction ${ }^{23}$ ), the $\gamma \alpha$ value has to be equal to half of this value. As seen from Fig. I7(b) the agreement is satisfactory with both the cases at $\sigma<(10-15) G_{0}$ if one takes into account the experimental error. At $\sigma \simeq 30 G_{0}$ the difference becomes crucial.

The discrepancy between the data obtained in different manner evident in Figs. 7(a) and 7(b) at high conductivity probably means that our assumption about smallness of the correction to the Hall conductivity $\sigma_{x y}$ is not valid in this case suggesting further investigations are needed to understand the origin of the low field anomaly in the Hall effect in the relatively clean systems.

Thus, the second order correction $\delta \sigma_{x x}^{\mathrm{WL} \times \mathrm{I}}$ caused by interplay between the WL and interaction corrections is of importance in our case. At low conductivity, $\sigma<$ $(10-15) G_{0}$, this correction contributes to the diagonal component of the conductivity tensor $\sigma_{x x}$ only. Its temperature dependence is metallic like in contrast to the WL and AA corrections which are insulating. Its magnetic field dependence is close in the shape to that of the WL correction, although the magnetoconductivity itself is negative in contrast to that induced by suppression of the weak localization. Existence of this correction results in: (i) the depressing of the interference induced low magnetic field magnetoresistance; (ii) the difference between the slope of the $\sigma$ vs $\ln T$ dependence and the value of $1+K_{e e}^{\mathrm{AA}}$; (iii) the occurrence of the beak in the $R_{H}$ vs $B$ dependence in low magnetic field. 


\section{CONCLUSION}

We have studied the nonlinear behavior of the Hall resistivity in the vicinity of zero magnetic field. Investigating the two-dimensional electron gas in strongly disordered $\mathrm{GaAs} / \mathrm{In}_{x} \mathrm{Ga}_{1-x} \mathrm{As} / \mathrm{GaAs}$ quantum well we have shown that the anomaly of the Hall resistance and impossibility of description of the temperature dependences of zero-field conductivity by taking into account only two first order WL and AA quantum corrections are explained by significant contribution of the second order correction resulting from the effect of weak localization on the interaction correction and vice versa in disordered systems with $\sigma<(10-15) G_{0}$. The experimental results are satisfactorily interpreted under assumption that this correction contributes to the diagonal component of the conductivity tensor $\sigma_{x x}$ only, its magnetic field dependence is close to that of the weak localization correction, although the temperature dependence is metallic-like.

\section{Acknowledgments}

We would like to thank I. S. Burmistrov and I. V. Gornyi for illuminating discussions. This work has been supported in part by the RFBR (Grant Nos 08-0200662, 09-02-00789, and 10-02-00481).
1 We consider the effects caused by the Landau quantization as being beyond the topic of this paper.

2 B. L. Altshuler and A. G. Aronov, in Electron-Electron Interaction in Disordered Systems, edited by A. L. Efros and M. Pollak (North Holland, Amsterdam, 1985) p. 1.

3 I. L. Aleiner, B. L. Altshuler, and M. E.Gershenzon, Waves Random Media, 9, 201 (1999).

4 G. Zala, B. N. Narozhny, and I. L. Aleiner, Phys. Rev. B, 64,214204 (2001).

5 I. V. Gornyi and A. D. Mirlin, Phys. Rev. Lett., 90, 076801 (4003).

6 I. V. Gornyi and A. D. Mirlin, Phys. Rev. B, 69, 045313 (2004).

7 D. A. Poole, M. Pepper, and R. W. Glew, J. Phys C: Solid State Phys, 14, L995 (1981).

8 D. J. Newson, M. Pepper, E. Y. Hall, and G. Hill, J. Phys. C: Solid State Phys, 20, 4369 (1987).

9 E. Tousson and Z. Ovadyahu, Phys. Rev. B, 38, 12290 (988).

10 G. M. Minkov, A. V. Germanenko, O. E. Rut, A. A. Sherstobitov, V. A. Larionova, A. K. Bakarov, and B. N. Zvonkov, Phys. Rev. B, 74, 045314 (2006).

11 A. Y. Kuntsevich and V. M. Pudalov, private communication.

12 V. A. Tkachenko and Z. D. Kvon, private communication.

13 Generally, the simultaneous existence of the $B$ dependent WL correction and the interaction correction to $\sigma_{x x}$, which is independent of the magnetic field, should lead to the low magnetic field dependence of $R_{H}$. However, this effect is the next order of smallness. Moreover, the Hall coefficient should decrease in magnitude with the $B$ increase if the sign of the interaction correction is insulating. The experimental behavior is opposite.

14 Analyzing the data we will neglect the interaction corrections in the Cooper channel. Two terms in low magnetic fields contribute to the low-magnetic field magnetoconductance. They are Maki-Thomson correction and correction to the density of states (DoS). The role of these terms in the low field magnetoconductivity is thoroughly considered in Ref. 23. We mention only that the DoS term contributes to $\sigma_{x x}$ and do not to $\sigma_{x y}$ as well as AA correction, but in contrast to it the DoS term yields the magnetoconductivity close in the shape to that of interference induced magnetoconductivity. However, our estimations show that the DoS correction is three-to-five times smaller in magnitude than the second-order corrections under our experimental conditions. They results in about $5-7 \%$ depth of the beak in the Hall effect instead of $\sim 30 \%$ observed experimentally.

15 A. M. Finkel'stein, Zh. Eksp. Teor. Fiz., 84, 168 (1983), [Sov. Phys. JETP 57, 97 (1983)].

16 A. M. Finkel'stein, Z. Phys. B: Condens. Matter, 56, 189 (1984).

17 C. Castellani, C. Di Castro, P. A. Lee, and M. Ma, Phys. Rev. B, 30, 527 (1984).

${ }^{18}$ C. Castellani, C. Di Castro, P. A. Lee, M. Ma, S. Sorella, and E. Tabet, Phys. Rev. B, 30, 1596 (1984).

19 C. Castellani, C. Di Castro, and P. A. Lee, Phys. Rev. B, 57, R9381 (1998)

A. P. Dmitriev, V. Y. Kachorovskii, and I. V. Gornyi, Phys. Rev. B, 56, 9910 (1997).

21 S. Hikami, A. I. Larkin, and Y. Nagaoka, Prog. Theor. Phys., 63, 707 (1980).

22 H.-P. Wittmann and A. Schmid, J. Low Temp. Phys, 69, 131 (1987).

${ }^{23}$ G. M. Minkov, A. V. Germanenko, and I. V. Gornyi, Phys. Rev. B, 70, 245423 (2004).

${ }^{24}$ G. M. Minkov, A. V. Germanenko, O. E. Rut, A. A. Sherstobitov, and B. N. Zvonkov, Phys. Rev. B, 79, 235335 (2009).

A. M. Finkel'stein, "Electron liquid in disordered conductors," in Electron-Electron Interaction in Disordered Systems, Vol. 14, edited by I. M. Khalatnikov (Harwood, London, 1990).

26 G. M. Minkov, A. V. Germanenko, O. E. Rut, A. A. Sherstobitov, and B. N. Zvonkov, Phys. Rev. B, 79, 235335 (2009).

27 A. M. Finkel'stein, Zh. Eksp. Teor. Fiz., 86, 367 (1984), [Sov. Phys. JETP 59, 212 (1984)].

28 R. Raimondi, C. Castellani, and C. Di Castro, Phys. Rev. B, 42, 4724 (1990).

${ }^{29}$ G. M. Minkov, A. V. Germanenko, O. E. Rut, A. A. Sherstobitov, and B. N. Zvonkov, Phys. Rev. B, 76, 165314 (2007).

30 S. Hikami, Phys. Rev. B, 24, 2671 (1981).

31 F. Wegner, Z. Phys. B: Condens. Matter, 35, 207 (1979).

32 F. Wegner, Phys. Rep., 67, 15 (1980).

${ }^{33}$ F. Wegner, Nucl. Phys. B, 316, 663 (1989). 
${ }^{34}$ M. A. Baranov, I. S. Burmistrov, and A. M. M. Pruisken. ${ }^{36}$ B. Shapiro and E. Abrahams, Phys. Rev. B, 24, 4025 Phys. Rev. B, 66, 075317 (2002). (1981).

35 A. Punnoose and A. M. Finkel'stein, Science, 310, 289 ${ }^{37}$ I. V. Gornyi and S. G. Novokshonov, private communica(2005). tion. 